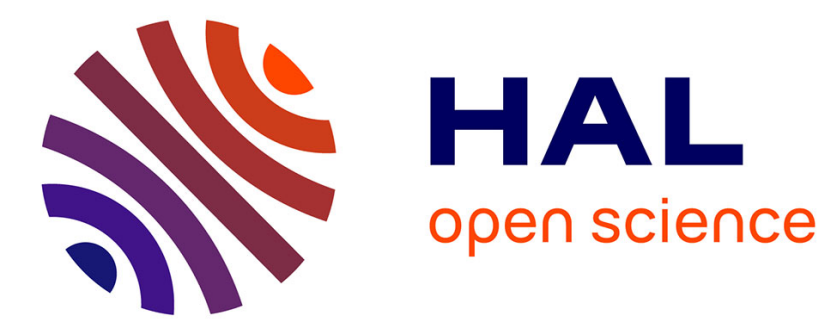

\title{
RECONSTRUCTION DE FRONTS D'ONDE DISTORDUS PAR LA TURBULENCE
}

Ph. Blanc-Benon, Y. Hugon-Jeannin, D. Juve

\section{To cite this version:}

Ph. Blanc-Benon, Y. Hugon-Jeannin, D. Juve. RECONSTRUCTION DE FRONTS D'ONDE DISTORDUS PAR LA TURBULENCE. Journal de Physique IV Proceedings, 1992, 02 (C1), pp.C1-545C1-548. 10.1051/jp4:19921117 . jpa-00251072

\section{HAL Id: jpa-00251072 https://hal.science/jpa-00251072}

Submitted on 1 Jan 1992

HAL is a multi-disciplinary open access archive for the deposit and dissemination of scientific research documents, whether they are published or not. The documents may come from teaching and research institutions in France or abroad, or from public or private research centers.
L'archive ouverte pluridisciplinaire HAL, est destinée au dépôt et à la diffusion de documents scientifiques de niveau recherche, publiés ou non, émanant des établissements d'enseignement et de recherche français ou étrangers, des laboratoires publics ou privés. 


\title{
RECONSTRUCTION DE FRONTS D'ONDE DISTORDUS PAR LA TURBULENCE
}

\author{
Ph. BLANC-BENON, Y. HUGON-JEANNIN et D. JUVE \\ Laboratoire de Mécanique des Fluides et d'Acoustique, CNRS URA-263, Ecole Centrale de Lyon, \\ BP. 163, F-69131 Ecully cedex, France
}

\begin{abstract}
The wave front distortions of a spherical wave that propagates through a turbulent thermal field are investigated under a variety of propagation conditions. Our measurements of phase difference demonstrate the limitation of the Rytov's method and point out some difficulties related to the occurence of random caustics in the saturation region.
\end{abstract}

\section{1 - INTRODUCTION}

En se propageant sur de longues distances dans un milieu turbulent les ondes acoustiques subissent des perturbations importantes dues aux fluctuations aléatoires du milieu. Les modifications des caractéristiques spatio-temporelles de l'onde transmise, associées aux fluctuations de phase et d'amplitude, se traduisent en particulier par une déformation des fronts d'onde et une perte de cohérence entre les capteurs d'une antenne ce qui en limite le pouvoir de résolution. Nous décrivons ici une expérience de propagation acoustique à travers une turbulence thermique. Les résultats présentés concernent l'évolution temporelle des fronts de phase reconstitués à partir d'une antenne de huit microphones et leur déformation progressive en fonction du régime de propagation (existence ou non de trajets multiples) ainsi que l'estimation de la fonction de structure des fluctuations de phase.

\section{2 - INSTALLATION EXPERIMENTALE}

Pour que les mesures effectuées soient représentatives des essais de propagation réalisés dans l'atmosphère, il est nécessaire que la distance de propagation $x$ dans la turbulence soit grande devant l'échelle de corrélation spatiale $\mathrm{L}_{\mathrm{T}}$ des fluctuations de température, et que la longueur d'onde acoustique $\lambda$ soit petite vis-à-vis de cette même échelle $L_{T}$. Pour l'installation réalisée dans la chambre anéchoïde de I'ECL $(10 \mathrm{~m} \times 8 \mathrm{~m} \times 7 \mathrm{~m})$ le volume de turbulence est créé par un réseau de résistances électriques fonctionnant en convection libre (Fig. 1). Après mélange des panaches thermiques, les fluctuations de température sont de $5^{\circ} \mathrm{C}$ dans le plan $\mathrm{H}=180 \mathrm{~cm}$ et l'échelle intégrale $\mathrm{L}_{\mathrm{T}}$ est de $7,6 \mathrm{~cm}$ [1]. Les sources acoustiques sont des émetteurs ultrasonores quasi-sphériques dont la fréquence d'émission est comprise entre $20 \mathrm{kHz}$ et $100 \mathrm{kHz}$. La distance de propagation $x$ varie de $0,5 \mathrm{~m}$ à $4 \mathrm{~m}$. Les signaux de pression acoustique sont mesurés avec huit microphones Bruël \& Kjaër 4135. Un traitement numérique des signaux permet de repérer tous les passages à zéro sur chaque voie de l'antenne pour des échantillons de $64 \mathrm{Kmots}$ et d'une durée de $5,25 \mathrm{~s}$. En choisissant une voie de l'antenne comme référence on déduit alors les phases instantanées entre les différentes voies j soient $\Delta \phi^{1 j}(2 \leq j \leq 8)$ [2]. Afin de caractériser de façon plus précise les différents régimes de propagation nous utilisons les paramètres $\Lambda$ et $\phi$ définis par [3]: $\Lambda=x / \mathrm{k}_{0} \mathrm{~L}_{\mathrm{T}}^{2}$ et $\phi^{2}=2 \mathrm{~L}_{\mathrm{T}} \mathrm{k}_{0}^{2} \times\left(\sqrt{\mathrm{T}^{2}} n \frac{2}{\mathrm{~T}}\right)^{2}$. En traçant les courbes (Fig. 2) $\phi=1, \Lambda \phi^{4,2}=1$ et $\Lambda \phi^{2,4}=1$ on détermine trois régions : la zone de saturation, la zone de validité de la solution de Rytov et une zone intermédiaire où les fluctuations d'intensité de l'onde sont dites partiellement saturées [4]. Dans cette représentation des phénomènes, les régions de saturation et saturation partielle correspondent à l'existence de plusieurs trajets possibles entre une source et un récepteur[3]. 


\section{RESULTATS}

a) Propagation sans saturation : pour une distance $x$ de $0,5 \mathrm{~m}$ les essais effectués avec une source sphérique émettant à $23,5 \mathrm{kHz}$ correspondent à un régime où la solution de Rytov est valable. Sur la figure 3 nous donnons un exemple d'évolution des écarts de phase instantanés $\Delta \phi^{1 j}$ entre la voie 1 de l'antenne et chacune des autres voies pour un temps d'observation assez long de 5,25 s. On observe des variations importantes de phase qui peuvent atteindre $2 \pi$ sur des durées assez courtes de l'ordre de quelques millisecondes lorsque l'espacement $\rho$ entre les microphones devient important ( $\rho$ $=14 \mathrm{~cm}$ pour $\Delta \phi^{18}$ ). A partir de ces écarts nous pouvons tracer l'évolution des fronts d'onde le long de l'antenne. Sur la figure 4 nous avons extrait de l'échantillon précédent six fronts espacés en temps de $0,1 \mathrm{~s}$ à partir de l'instant $t=1,9 \mathrm{~s}$. L'aspect fripé de ces fronts d'onde traduit la scintillation angulaire de la source acoustique visée par l'antenne à travers le milieu turbulent. Pour estimer cette scintillation nous avons calculé la variance des fluctuations de phase entre voies et nous l'avons comparé aux estimations théoriques de la fonction de structure des fluctuations de phase $\mathrm{D}_{\mathrm{S}}$ obtenues par la solution de Rytov [5]. En modélisant les fluctuations d'indice $\varepsilon=-T^{\prime} / \bar{T}$ à partir d'un spectre de von Karman $\phi_{\varepsilon}(K)$ on montre que $D_{S}(x, \rho)$ se met sous la forme:

$$
\begin{gathered}
D_{s}(x, \rho)=8 \pi^{2} \int_{0}^{x} \mathrm{dz} \int_{0}^{\infty} \mathrm{K}\left(1-\mathrm{J}_{0}\left(\mathrm{~K} \frac{z}{\mathrm{x}} \rho\right)\right) \mathrm{k}_{0}^{2} \cos ^{2}\left(\frac{z(\mathrm{x}-\mathrm{z})}{2 \mathrm{k}_{0} \mathrm{x}}\right) \phi_{\varepsilon}(\mathrm{K}) \mathrm{dK} \\
\phi_{\varepsilon}(\mathrm{K})=0,033 \mathrm{C}_{\varepsilon}^{2}\left(\mathrm{~K}^{2}+\frac{1}{\mathrm{~L}_{0}^{2}}\right)-11 / 6 \exp \left(-\frac{\mathrm{k}^{2}}{\mathrm{Km}^{2}}\right) ; \mathrm{C}_{\varepsilon}^{2}=1,91\left(\sqrt{\overline{\mathrm{T}^{\prime}}} / 2 \overline{\mathrm{T}}\right)^{2} \mathrm{~L}_{0}^{-2 / 3} ; \mathrm{L}_{0}=1,339 \mathrm{~L}_{\mathrm{T}} ; \mathrm{Km}=5,92 \Omega_{0}
\end{gathered}
$$

Dans nos expériences $L_{0}=10,17 \mathrm{~cm}$ et $\mathrm{l}_{0}=0,1 \mathrm{~cm}$. L'accord entre les mesures et la solution de Rytov est tout à fait satisfaisant (Fig. 5).

b) Propagation avec saturation : en plaçant l'antenne acoustique à $1 \mathrm{~m}$ d'une source émettant à $23,5 \mathrm{kHz}$ on commence à voir apparaître des fluctuations de phase entre voies nettement supérieures à $2 \pi$ qui se traduisent par un décalage de phase et une modification de la valeur moyenne par bloc de traitement (Fig. 6). Sur les figures 7 et 8 nous avons tracé les signaux de pression acoustique correspondant aux voies 1,6 et 7 . On constate que les voies 1 et 6 sont dabord en phase pour $t=1,175 \mathrm{~s}$ puis en opposition de phase pour $t \approx 1,183 \mathrm{~s}$ et de nouveau en phase pour $t>1,187 \mathrm{~s}$. Pour les voies 1 et 7 l'amplitude s'annule pour $\mathrm{t}=1,187 \mathrm{~s}$ et dans un intervalle de temps inférieur à une période du signal de la voie 1 , la voie 7 devient en opposition de phase. Ces transitions sont continues. Elles s'expliquent vraisemblablement par la formation aléatoire de zones de focalisation de l'onde. On note alors une perte de référence de phase des microphones de l'antenne suivant leur position par rapport aux lieux géométriques de positionnement des caustiques ([2], [5]). Pour ces cas la solution de Rytov ne permet plus d'estimer la variance des fluctuations de phase entre les différentes voies de l'antenne et de prendre en compte ces variations continues de phase supérieures à $\pi$.

\section{4-CONCLUSION}

Les mesures effectuées nous ont permis d'étudier l'influence du régime de propagation dans la turbulence (saturé ou non saturé) sur la déformation des surfaces équiphases et la limitation de la méthode de Rytov. De plus, nous avons mis en évidence l'existence de variations continues de phases supérieures à $\pi$ et l'importance de la focalisation aléatoire du milieu sur la déformation des fronts d'onde.

\section{Les auteurs remercient la DRET pour son soutien financier.}

\section{REFERENCES}

(1) BLANC-BENON Ph., (1987), These Docteur-ès-Sciences, UCB LYON I, n $87-49$.

[2] BLANC-BENON Ph. et al., (1988), Rapport Final contrat DRET 88.488.

[3] FLATTE S.M. et al., (1979), Cambridge Press N.Y.

[4] BLANC-BENON Ph. et JUVE D., (1990), Colloque de Physique Tome 51, C3-13-22.

[5] TATARSKI V.I., (1991), IPST, IPST Keter Press Jenusalem.

[6] BLANC-BENON Ph., JUVE D. et COMTE-BELLOT G.. (1991), Theoret. Comput. Fluid Dynamics 2, 271-278. 


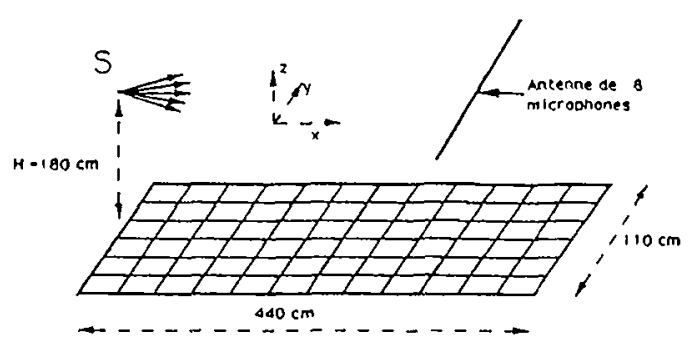

Fig. I : Schéma de l'installation expérimentale

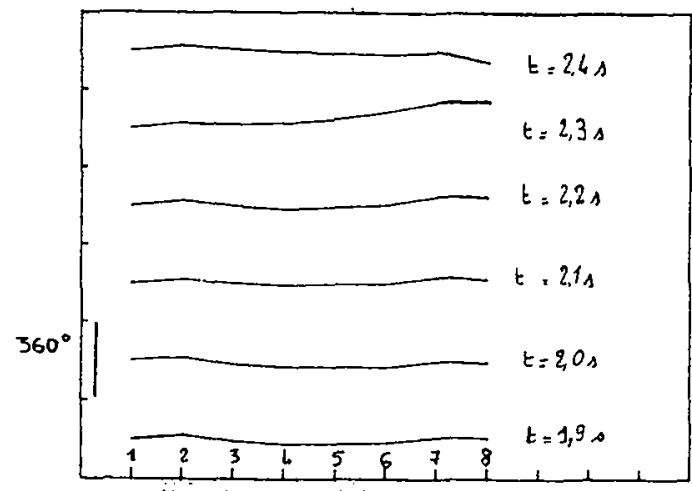

Mierophone de réffrence 1

Fig. 4 : Evolution temporelle des surfaces équi-phases reconstituées à partir d'une antenne de 8 microphones $(f=23,5 \mathrm{kHz} ; x=0,5 \mathrm{~m}$ )

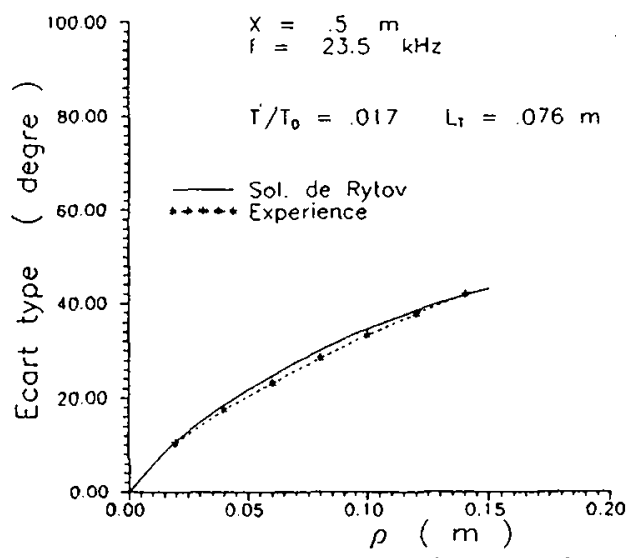

Fig. 5 : Ecart type des fluctuations de phase entre voies pour différentes séparations $\rho$ entre les microphones. Comparaison avec la fonction de structure therorique de la solution de Rytov

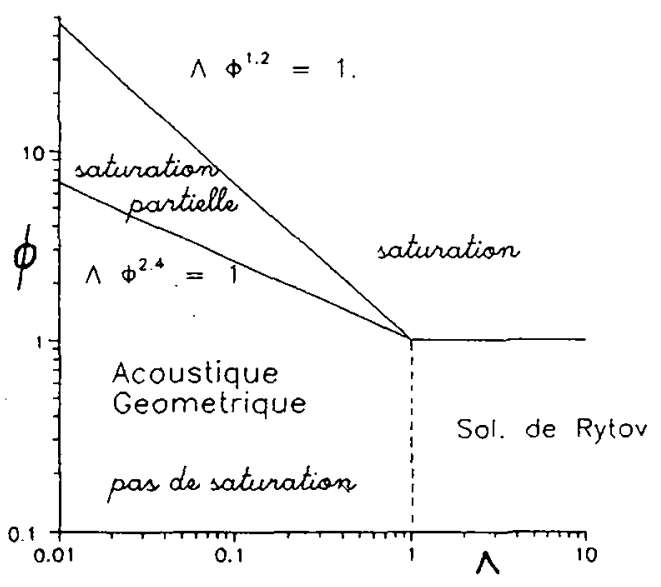

Fig. 2 : Diagramme $\phi \cdot A$

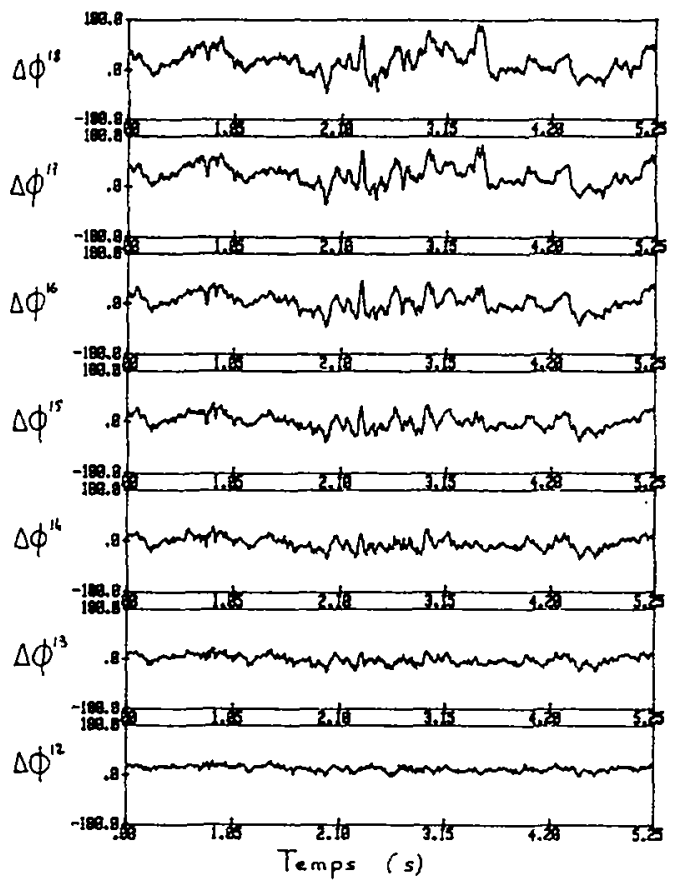

Fig. 3 : Ecart de phase instantanée entre les yoies de l'antenne

(Reference voie $I$

$f=23,5 \mathrm{kHz}, \quad x=0,5 \mathrm{~m}$ ) 


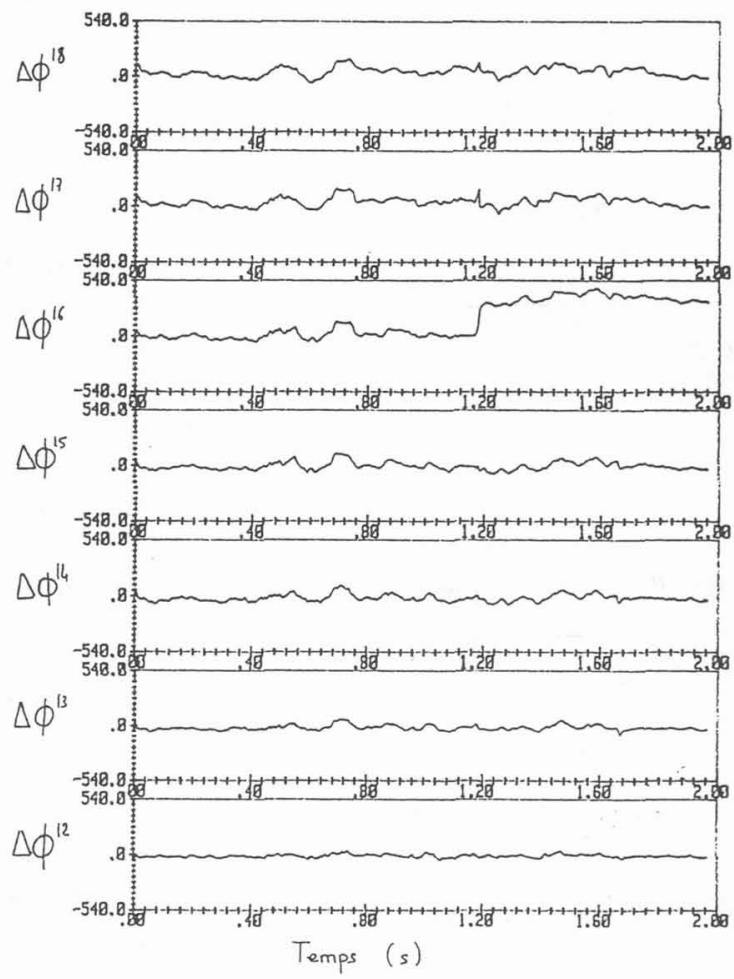

Fig. 6 : Ecart de phase instantanée entre les voies de l'antenne (Référence voie $1, f=23,5 \mathrm{kHz}, x=1 \mathrm{~m}$ )

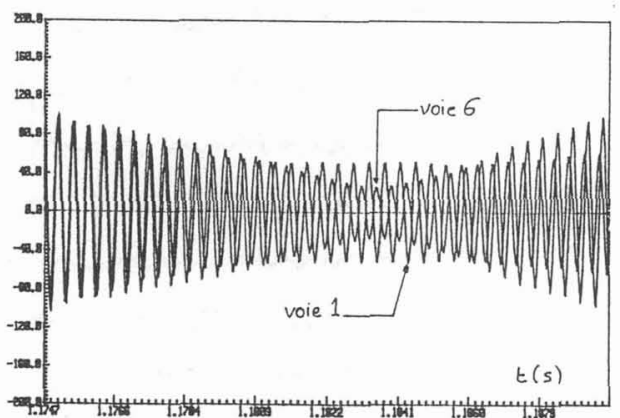

Fig. 7 : Visualisation des signaux de pression (yoies 1 et 6) rumérisés pour $t$ compris entre $1,17 \mathrm{~s}$ et $1,19 \mathrm{~s}$. Illustration de la variation continue de la phase

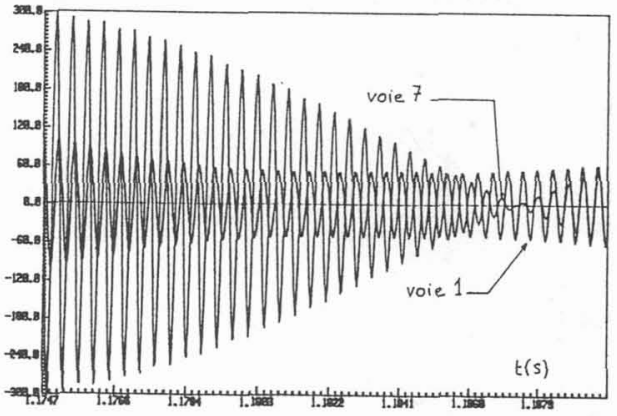

Fig. 8 : Visualisation des signaux de pression (voies $I$ et 7) numérisés pour $t$ compris entre $1,17 s$ et 1,19 s. Illustration de la variation continue de la phase 